CUBO A Mathematical Journal

Vol.14, No 02, (153-173). June 2012

\title{
Local energy decay for the wave equation with a time-periodic non-trapping metric and moving obstacle
}

\author{
YAVAR KIAN \\ Centre de Physique Théorique CNRS-Luminy, \\ Case 907, 13288 Marseille, France. \\ email: Yavar.Kian@cpt.univ-mrs.fr
}

\begin{abstract}
Consider the mixed problem with Dirichelet condition associated to the wave equation $\partial_{t}^{2} \mathfrak{u}-\operatorname{div}_{x}\left(a(t, x) \nabla_{x} u\right)=0$, where the scalar metric $a(t, x)$ is T-periodic in $t$ and uniformly equal to 1 outside a compact set in $x$, on a T-periodic domain. Let $\mathcal{U}(t, 0)$ be the associated propagator. Assuming that the perturbations are non-trapping, we prove the meromorphic continuation of the cut-off resolvent of the Floquet operator $\mathcal{U}(\mathrm{T}, 0)$ and we establish sufficient conditions for local energy decay.
\end{abstract}

\section{RESUMEN}

Considere el problema mixto con condiciones de Dirichlet asociadas a la ecuación de onda $\partial_{t}^{2} u-\operatorname{div}_{x}\left(a(t, x) \nabla_{x} u\right)=0$, donde la métrica escalar $a(t ; x)$ es T-periódica en $t$ y uniformemente igual a 1 fuera de un conjunto compacto en $x$, sobre un dominio T-periódico. Sea $\mathcal{U}(t, 0)$ el propagador asociado. Asumiendo que las perturbaciones son non-trapping, probamos la continuación meromorfa de la resolvente de corte del operador de Floquet $\mathcal{U}(\mathrm{T}, 0)$ y establecemos condiciones suficientes para la decadencia local de energía.

Keywords and Phrases: time-dependent perturbation, moving obstacle, local energy decay, wave equation.

2010 AMS Mathematics Subject Classification: 35B40, 35L15 . 


\section{Introduction}

Let $\Omega$ be an open domain in $\mathbb{R}^{1+n}, n \geq 3$ with $\mathcal{C}^{\infty}$ boundary $\partial \Omega$. Introduce the sets

$$
\Omega(t)=\left\{x \in \mathbb{R}^{n}:(t, x) \in \Omega\right\}, \quad O(t)=\mathbb{R}^{n} \backslash \Omega(t), \quad t \in \mathbb{R} .
$$

We assume that there exists $\rho_{1}>0$ such that for all $t \in \mathbb{R}$

$$
\mathrm{O}(\mathrm{t}) \subset\left\{x:|x| \leq \rho_{1}\right\}
$$

Moreover there exists $\mathrm{T}>0$ such that

$$
\mathrm{O}(\mathrm{t}+\mathrm{T})=\mathrm{O}(\mathrm{t}), \quad \mathrm{t} \in \mathbb{R}
$$

For each $(t, x) \in \partial \Omega$, let $v(t, x)=\left(v_{t}(t, x), v_{x}(t, x)\right)$ be the exterior unit normal vector to $\partial \Omega$ at $(t, x) \in \partial \Omega$ pointing into $\Omega$. Then, we assume that there exists $0<c<1$ such that

$$
\left|v_{\mathrm{t}}\right|<\mathrm{c}\left|v_{\mathrm{x}}\right|
$$

Consider the following mixed problem

$$
\left\{\begin{aligned}
\mathfrak{u}_{t t}-\operatorname{div}_{x}\left(a(t, x) \nabla_{x} u\right) & =0, \quad(t, x) \in \Omega \\
u_{\mid \partial \Omega} & =0 \\
\left(u, u_{t}\right)(s, x)=\left(f_{1}(x), f_{2}(x)\right) & =f(x), \quad x \in \Omega(s)
\end{aligned}\right.
$$

where the perturbation $a(t, x) \in \mathcal{C}^{\infty}\left(\mathbb{R}^{n+1}\right)$ is a scalar function which satisfies the conditions:

(i) $C \geq a(t, x) \geq c>0,(t, x) \in \mathbb{R}^{n+1}$,

(ii) there exists $\rho>\rho_{1}$ such that $a(t, x)=1$ for $|x| \geq \rho$,

(iii) there exists $T>0$ such that $a(t+T, x)=a(t, x),(t, x) \in \mathbb{R}^{n+1}$.

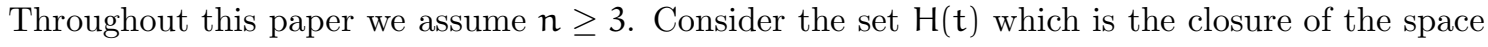
$\mathcal{C}_{0}^{\infty}(\Omega(t)) \times \mathcal{C}_{0}^{\infty}(\Omega(t))$ with respect to the norm

$$
\|f\|_{H(t)}=\left(\int_{\Omega(t)}\left(\left|\nabla_{x} f_{1}\right|^{2}+\left|f_{2}\right|^{2}\right) d x\right)^{\frac{1}{2}}, \quad f=\left(f_{1}, f_{2}\right) \in \mathcal{C}_{0}^{\infty}(\Omega(t)) \times \mathcal{C}_{0}^{\infty}(\Omega(t)) .
$$

Let us introduce some general properties of solutions of (1.4). We show, in Section 1, that for $f \in H(s)$ there exists a unique solution of (1.4) and we introduce the propagator

$$
\mathcal{U}(t, s): H(s) \ni\left(f_{1}, f_{2}\right)=f \mapsto \mathcal{U}(t, s) f=\left(u, u_{t}\right)(t, x) \in H(t)
$$

with $u$ the solution of (1.4). Moreover, we prove that $\mathcal{U}(t, s)$ is a bounded operator satisfying the following estimate

$$
\|\mathcal{U}(\mathrm{t}, \mathrm{s})\|_{\mathcal{L}(\mathrm{H}(\mathrm{s}), \mathrm{H}(\mathrm{t}))} \leq \mathrm{B} e^{\mathrm{A}|\mathrm{t}-\mathrm{s}|} .
$$


The goal of this paper is to establish sufficient conditions for a local energy decay taking the form

$$
\|\chi \mathcal{U}(\mathrm{t}, \mathrm{s}) \chi\|_{\mathcal{L}(\mathrm{H}(\mathrm{s}), \mathrm{H}(\mathrm{t}))} \leq \mathrm{C}_{\chi} \mathrm{p}(\mathrm{t}-\mathrm{s}), \quad \mathrm{t} \geq \mathrm{s},
$$

with $p(t) \in \mathrm{L}^{1}\left(\mathbb{R}^{+}\right)$and $\chi \in \mathcal{C}_{0}^{\infty}(|x| \leq \rho+1)$.

We study problem (1.4) under a non-trapping condition. More precisely, let $\mathcal{U}\left(t, s, x, x_{0}\right)$ be the kernel of the propagator $\mathcal{U}(t, s)$ and consider the following

(H1) For all $r>0$, there exists $T_{1}(r)>0$ such that

$$
\mathcal{U}\left(t, s, x, x_{0}\right) \in \mathcal{C}^{\infty}\left(\left\{\left(t, s, x, x_{0}\right):|x| \leq r,\left|x_{0}\right| \leq r,|t-s| \geq T_{1}(r)\right\}\right) .
$$

From [15], we know that singularities propagate along null-bicharacteristics (with consideration of their reflections from $\partial \Omega$ ). Thus, one can show that condition (H1) is equivalent to the requirement that all null-bicharacteristics of (1.4) with consideration of reflections from $\partial \Omega$ go out to infinity as $|t-s| \rightarrow+\infty$. Let us recall that the non-trapping condition (H1) is necessary for (1.8) since for some trapping perturbations we may have solutions with exponentially increasing energy (see [7] for $\Omega=\mathbb{R}^{1+n}$ and 22 for $\left.a(t, x)=1\right)$. On the other hand, even for non-trapping periodic perturbations some parametric resonances could lead to solutions with exponentially growing energy (see [6] for time-periodic potentials). To exclude the existence of such solutions we must consider a second assumption.

Many authors have investigated the local energy decay of wave equations. The main hypothesis is that the perturbations are non-trapping. For $a(t, x)=a_{0}(x)$ independent of time and fixed obstacles, the meromorphic continuation and estimates of the cut-off resolvent $\chi\left(-\operatorname{div}_{\chi}\left(a_{0}(x) \nabla_{\chi} \cdot\right)-\lambda^{2}\right)^{-1} \chi$, where $\chi \in \mathcal{C}_{0}^{\infty}\left(\mathbb{R}^{n}\right)$ and $\lambda \in \mathbb{C}$, are the main arguments for estimate (1.8) (see [24], 25], [27] and [28]). From these results, by considering the connection between the Fourier transform in time of the solutions and the stationary operator $-\operatorname{div}_{x}\left(a_{0}(x) \nabla_{x}.\right)-\lambda^{2}$, one can deduce (1.8). For time dependent metric $a(t, x)$ or moving obstacle, since the domain or the Hamiltonian $-\operatorname{div}_{x}\left(a(t, x) \nabla_{x}\right.$. $)$ are time-dependent, we cannot apply these arguments. However, the analysis of the Floquet operator $\mathcal{U}(\mathrm{T}, 0)$ makes it possible to obtain (1.8) with $\mathrm{T}$-periodic perturbations and moving obstacle. In [8] the authors have extended the Lax-Phillips theory to problem (1.4) with $a(t, x)=1$ and they have established a local energy decay (1.8). By using the compactness of the local evolution operator, deduced from a propagation of singularities, and the RAGE theorem of Georgiev and Petkov (see [9]), Bachelot and Petkov have shown in [1] that in the case of odd dimensions, the decay of the local energy associated to the wave equation with time periodic potential is exponential for initial data with compact support included in a subspace of finite codimension. Petkov has extended this result to even dimensions (see [21]), by using the meromorphic continuation of the cut-off resolvent of the Floquet operator associated to this problem.

Let us introduce the cut-off resolvent, associated to the Floquet operator $\mathcal{U}(\mathrm{T}, 0)$, defined by

$$
R_{\psi_{1}, \psi_{2}}(\theta)=\psi_{1}\left(\mathcal{U}(T, 0)-e^{-i \theta}\right)^{-1} \psi_{2}: H(0) \rightarrow H(0), \quad \psi_{1}, \psi_{2} \in \mathcal{C}_{0}^{\infty}\left(\mathbb{R}^{n}\right) .
$$


According to (1.7), $R_{\psi_{1}, \psi_{2}}(\theta)$ is a family of bounded operators analytic with respect to $\theta$ on $\{\theta \in \mathbb{C}: \mathfrak{I}(\theta)>A T\}$. Applying some arguments of [26], in Section 2, we show the meromorphic continuation of $R_{\psi_{1}, \psi_{2}}(\theta)$ to $\mathbb{C}$ for $n$ odd and to $\left\{\theta \in \mathbb{C}: \theta \notin 2 \pi \mathbb{Z}+i \mathbb{R}^{-}\right\}$for $n$ even. Let us recall that the meromorphic continuation of $R_{\psi_{1}, \psi_{2}}(\theta)$ is closely related to the asymptotic expansion of $\chi \mathcal{U}(t, 0) \chi, \chi \in \mathcal{C}_{0}^{\infty}\left(\mathbb{R}^{n}\right)$, as $t \rightarrow+\infty$ (see Section 2 and the main theorem in [26]). Consequently, it seems natural to consider the meromorphic continuations of $R_{\psi_{1}, \psi_{2}}(\theta)$ that imply (1.8). Consider the following assumption.

(H2) There exist $\phi_{1}, \phi_{2} \in \mathcal{C}_{0}^{\infty}\left(\mathbb{R}^{n}\right)$, satisfying $\phi_{1}(x)=\phi_{2}(x)=1$ for $|x| \leq \rho+T+2$, such that the operator $R_{\phi_{1}, \phi_{2}}(\theta)$ admits an analytic continuation from $\{\theta \in \mathbb{C}: \operatorname{Im}(\theta) \geq A>0\}$ to $\{\theta \in \mathbb{C}: \operatorname{Im}(\theta) \geq 0\}$, for $n \geq 3$, odd, and to $\{\theta \in \mathbb{C}: \operatorname{Im}(\theta)>0\}$ for $n \geq 4$, even. Moreover, for $n$ even, $R_{\phi_{1}, \phi_{2}}(\theta)$ admits a continuous continuation from $\{\theta \in \mathbb{C}: \operatorname{Im}(\theta)>0\}$ to $\{\theta \in \mathbb{C}: \operatorname{Im}(\theta) \geq 0, \theta \neq 2 k \pi, k \in \mathbb{Z}\}$ and we have

$$
\limsup _{\substack{\lambda \rightarrow 0 \\ \operatorname{Im}(\lambda)>0}}\left\|R_{\phi_{1}, \phi_{2}}(\lambda)\right\|<\infty
$$

Assuming (H1) and (H2) fulfilled, we obtain the following.

Theorem 1. Assume (1.1), (1.2), (1.3), (1.5), (H1) and (H2) fulfilled. Then, estimate (1.8) is fulfilled with

$$
\left\{\begin{array}{l}
p(t)=e^{-\delta t} \text { for } n \geq 3 \text { odd } \\
p(t)=\frac{1}{(t+1) \ln ^{2}(t+e)} \text { for } n \geq 4 \text { even. }
\end{array}\right.
$$

Let us remark that, assuming (H1) fulfilled, (H2) is a necessary and sufficient condition for estimate (1.8) with $p(t)$ satisfying (1.9). Moreover, if (H2) is not fulfilled, even the uniform estimate in time of the local energy $\|\chi \mathcal{U}(t, 0) \chi\|_{\mathcal{L}(H(0), H(t))}$ may not hold. For example, if $R_{\phi_{1}, \phi_{2}}(\theta)$ has a pole $\theta_{0} \in \mathbb{C}$ with $\Im\left(\theta_{0}\right)>0$, one can establish the estimate

$$
\|\chi \mathcal{U}(t, 0) \chi\|_{\mathcal{L}(H(0), H(t))} \geq C e^{\frac{\Im\left(\theta_{0}\right)}{T} t}
$$

and deduce existence of a solution with compactly supported initial data and exponentially growing local energy. It has been established in [6] that these phenomenon can occur even with a nontrapping condition. The goal of (H2) is to avoid existence of such solutions.

Remark 1 . Let the metric $\left(a_{i j}(t, x)\right)_{1 \leq i, j \leq n}$ be such that for all $i, j=1 \cdots n$ we have

(i) there exists $\rho>0$ such that $a_{i j}(t, x)=\delta_{i j}$, for $|x| \geq \rho$, with $\delta_{i j}=0$ for $i \neq j$ and $\delta_{i i}=1$,

(ii) there exists $T>0$ such that $a_{i j}(t+T, x)=a_{i j}(t, x), \forall(t, x) \in \mathbb{R}^{n+1}$,

(iii) $a_{i j}(t, x)=a_{j i}(t, x), \forall(t, x) \in \mathbb{R}^{n+1}$,

(iv) there exist $C>c>0$ such that $C|\xi|^{2} \geq \sum_{i, j=1}^{n} a_{i j}(t, x) \xi_{i} \xi_{j} \geq c|\xi|^{2}, \quad \forall(t, x) \in \mathbb{R}^{1+n}, \xi \in \mathbb{R}^{n}$. 
If we replace $a(t, x)$ in (1.4) we get the following mixed problem

$$
\left\{\begin{aligned}
u_{t t}-\sum_{i, j=1}^{n} \frac{\partial}{\partial x_{i}}\left(a_{i j}(t, x) \frac{\partial}{\partial x_{j}} u\right) & =0, \quad(t, x) \in \Omega, \\
u_{\mid \partial \Omega} & =0, \\
\left(u, u_{t}\right)(s, x)=\left(f_{1}(x), f_{2}(x)\right) & =f(x), \quad x \in \Omega(s) .
\end{aligned}\right.
$$

All the results of this paper remain valid for the mixed problem (1.10) and their proofs follow from the same arguments.

Notice that the estimate

$$
\left\|\psi_{1} \mathcal{U}(\mathrm{NT}, 0) \psi_{2}\right\|_{\mathcal{L}(\mathrm{H}(0))} \leq \frac{\mathrm{C}_{\psi_{1}, \psi_{2}}}{(\mathrm{~N}+1) \ln ^{2}(\mathrm{~N}+e)}, \quad \mathrm{N} \in \mathbb{N},
$$

implies (1.8). On the other hand, if (1.11) is valid, the assumption (H2) for $n$ even is fulfilled. Indeed, for large $A>>1$ and $\operatorname{Im}(\theta) \geq A T$ we have

$$
R_{\psi_{1}, \psi_{2}}(\theta)=-e^{i \theta} \sum_{N=0}^{\infty} \psi_{1} \mathcal{U}(\mathrm{NT}, 0) \psi_{2} e^{i \mathrm{~N} \theta}
$$

and applying (1.11), we conclude that $R_{\psi_{1}, \psi_{2}}(\theta)$ admits an analytic continuation from $\{\theta \in \mathbb{C}: \operatorname{Im}(\theta) \geq A>0\}$ to $\{\theta \in \mathbb{C}: \operatorname{Im}(\theta)>0\}$. Moreover, $R_{\psi_{1}, \psi_{2}}(\theta)$ is bounded for $\theta \in \mathbb{R}$. In Section 4 , we give some examples of metrics $a(t, x)$ and moving obstacle $O(t)$ such that (1.11) is fulfilled.

\section{General properties}

The purpose of this section is to establish some general properties of solutions of problem (1.4). We will study the global well posedness of (1.4) and we will prove estimate (1.7). We start by fixing the notion of solutions of (1.4).

Definition 2.1. A distribution $u(t, x) \in \mathrm{D}^{\prime}(\Omega)$ is called a solution of (1.4) if the following conditions hold:

(i) $\left(\mathfrak{u}(t,),. \mathfrak{u}_{\mathfrak{t}}(t,).\right) \in H(t)$ for each $t \in \mathbb{R}$; extended inside $\mathrm{O}(\mathrm{t})$ by setting $\mathfrak{u}(\mathrm{t}, \mathrm{x})=0$, the functions

$$
\mathrm{t} \longmapsto \nabla_{\mathrm{x}} \mathrm{u}(\mathrm{t}, .), \quad \mathrm{t} \longmapsto \mathrm{u}_{\mathrm{t}}(\mathrm{t}, .)
$$

are continuous with values in $\mathrm{L}^{2}\left(\mathbb{R}^{n}\right)$, 
(ii) $\left(u(s,),. u_{t}(s,).\right)=\left(f_{1}, f_{2}\right)=f$

(iii) $\partial_{\mathfrak{t}}^{2} \mathfrak{u}-\operatorname{div}_{\chi}\left(a(t, x) \nabla_{x} u\right)=0$ in $\Omega$ in the sense of distributions.

In the next result we obtain the existence and uniqueness of solutions of (1.4).

Theorem 2. Assume (1.1), (1.2), (1.3) and (1.5) fulfilled. Then, for each $f \in H(s)$ there exists a unique solution $u(t,$.$) of (1.4) with the property that for each t>0$

$$
\sup _{\substack{|t-s| \leq D \\|s| \leq 2 D}}\left\|\left(u(t, .), u_{t}(t, .)\right)\right\|_{H(t)} \leq C_{D}\|f\|_{H(s)}
$$

Proof. First we treat the existence and uniqueness of the solution for small $|t-s|$. Given $z \in \Omega(s)$, consider the cone

$$
\mathrm{C}_{z, \mathrm{~s}}=\left\{(\mathrm{t}, \mathrm{x}) \in \mathbb{R}^{1+\mathrm{n}}:|x-z| \leq|\mathrm{t}-\mathrm{s}|\right\} .
$$

For $|t-s|$ small enough and for $z$ outside a small neighborhood of $\partial \Omega(s)$ we obtain $C_{z, s} \subset \Omega$. Consequently, for $(t, x) \in C_{z, s}$ the solution $\mathfrak{u}(t, x)$ of the mixed problem coincides with the solution of the Cauchy problem

$$
\left\{\begin{aligned}
\mathfrak{u}_{\mathrm{tt}}-\operatorname{div}_{x}\left(\mathfrak{a}(\mathrm{t}, x) \nabla_{x} \mathfrak{u}\right) & =0, \quad(t, x) \in \mathbb{R} \times \mathbb{R}^{n}, \\
\left(\mathfrak{u}, \mathfrak{u}_{\mathrm{t}}\right)(s, x)=\left(\mathrm{f}_{1}(x), f_{2}(x)\right) & =f(x), \quad x \in \mathbb{R}^{\mathrm{n}}
\end{aligned}\right.
$$

with $f$ extended by 0 for $x \in \mathrm{O}(\mathrm{s})$. Thus, for $|\mathrm{t}-\mathrm{s}| \leq \epsilon$ and $\epsilon$ sufficiently small, we will determine $u(t, x)$ in some small neighborhood of $\partial \Omega \cap\{|t-s| \leq \epsilon\}$. Given $(s, z)$ with $z \in \partial \Omega(s)$, we establish the existence and uniqueness of $u(t, x)$ in some space-time neighborhood of $(s, z)$. Covering the compact set $\{s\} \times \partial \Omega(s)$ by a finite number of such neighborhoods and using the local uniqueness result for the points where these neighborhoods overlap, we deduce the existence and uniqueness for small $|t-s|$. Introduce in a neighborhood of $(s, z), z \in \partial \Omega(s)$, local coordinates $(t, y), y^{\prime}=$ $\left(y_{1}, \ldots, y_{n-1}\right)$, so that $(s, z)$ is transformed into $(0,0)$, while the boundary $\partial \Omega$ is given by $y_{n}=$ $\mathrm{g}\left(\mathrm{t}, \mathrm{y}^{\prime}\right)$ with $\mathrm{g}$ a $\mathcal{C}^{\infty}$ function such that $\nabla_{y^{\prime}} \mathrm{g}(0,0)=0$. Since

$$
v\left(t, y^{\prime}, g\left(t, y^{\prime}\right)\right)=\frac{1}{\sqrt{1+\left|g_{t}\left(t, y^{\prime}\right)\right|^{2}+\left|\nabla_{y^{\prime}} g\left(t, y^{\prime}\right)\right|^{2}}}\left(-g_{t}\left(t, y^{\prime}\right),-\nabla_{y^{\prime}} g\left(t, y^{\prime}\right), 1\right),
$$

statement (1.3) implies that

$$
\left|g_{t}\left(t, y^{\prime}\right)\right|<c\left(\left|\nabla_{y^{\prime}} g\left(t, y^{\prime}\right)\right|+1\right) \text {. }
$$

Thus, we have $\left|g_{t}(0,0)\right|<c$. If we choose a sufficiently small neighborhood of $(0,0)$ we can assume that $\left|g_{t}\left(t, y^{\prime}\right)\right|<c$. Changing variables

$$
x_{j}=y_{j}, \quad j=1, \ldots, n-1, \quad x_{n}=y_{n}-g\left(t, y^{\prime}\right)
$$

we transform

$$
\partial_{t}^{2}-\operatorname{div}_{x}\left(a(t, x) \nabla_{x} \cdot\right)
$$


into the operator $\mathrm{P}\left(\mathrm{t}, x, \partial_{\mathrm{t}}, \partial_{x}\right)$ with principal symbol

$$
\begin{aligned}
\sigma\left(\mathrm{P}\left(\mathrm{t}, x, \partial_{\mathrm{t}}, \partial_{x}\right)\right)= & -\tau^{2}+2 \mathrm{~g}_{\mathrm{t}} \tau \xi_{\mathrm{n}}-2 \xi_{\mathrm{n}} \mathrm{b}(\mathrm{t}, \mathrm{x}) \xi^{\prime} \cdot \nabla_{x^{\prime}} \mathrm{g}+\mathrm{b}(\mathrm{t}, \mathrm{x})\left|\xi^{\prime}\right|^{2} \\
& +\left(\mathrm{b}(\mathrm{t}, x)\left|\nabla_{x^{\prime}} \mathrm{g}\right|^{2}-\mathrm{g}_{\mathrm{t}}^{2}+\mathrm{b}(\mathrm{t}, x)\right) \xi_{n}^{2},
\end{aligned}
$$

where $b(t, x)=a(t, y)$. Here $\left(\tau, \xi^{\prime}, \xi_{n}\right)$ are the variable dual to $\left(t, x^{\prime}, x_{n}\right)$. Statement (1.3) and property (1.5) imply that

$$
\mathrm{b}(\mathrm{t}, \mathrm{x})\left|\nabla_{x^{\prime}} \mathrm{g}\right|^{2}-\mathrm{gt}_{\mathrm{t}}^{2}+\mathrm{b}(\mathrm{t}, \mathrm{x})>0 .
$$

Consider the problem

$$
\left\{\begin{aligned}
\mathrm{P}\left(\mathrm{t}, x, \partial_{\mathrm{t}}, \partial_{x}\right) \mathfrak{u} & =0 \text { in } \mathbb{R}_{\mathrm{t}} \times \mathbb{R}_{x^{\prime}}^{\mathrm{n}-1} \times \mathbb{R}_{x_{n}}^{+}, \\
\mathfrak{u}\left(\mathrm{t}, x^{\prime}, 0\right) & =0 \text { in } \mathbb{R}_{\mathrm{t}} \times \mathbb{R}_{x^{\prime}}^{\mathrm{n}-1}, \\
\left(\mathfrak{u}(0, x), \mathfrak{u}_{\mathrm{t}}(0, x)\right) & =\mathrm{f}(x) .
\end{aligned}\right.
$$

We suitably extend the coefficients of $\mathrm{P}\left(\mathrm{t}, x, \partial_{t}, \partial_{x}\right)$ to $\mathbb{R}^{1+n}$ preserving the strict hyperbolicity of $\mathrm{P}\left(t, x, \partial_{t}, \partial_{x}\right)$ with respect to $t$. For the mixed problem (2.4) we can apply the results of Miyatake [18] and Hörmander [10, Chapter XXIV. Notice that the inequality (2.3) guarantees that the boundary $x_{n}=0$ is timelike in the sense of Hörmander [10. The result of Miyatake [18, says that if $\nabla_{x} f_{1}, f_{2} \in L_{\text {loc }}^{2}\left(\mathbb{R}_{\chi^{\prime}}^{n-1} \times \overline{\mathbb{R}_{x_{n}}^{+}}\right)$with $f_{1}=f_{2}$ for $x_{n}=0$, then for $|t| \leq \delta$ there exists a unique solution $\mathfrak{u}(\mathrm{t}, \mathrm{x}) \in \mathrm{H}_{\text {loc }}^{1}\left(\mathbb{R}_{\mathrm{x}^{\prime}}^{n-1} \times \overline{\mathbb{R}_{\mathrm{x}_{n}}^{+}}\right)$of (2.4) satisfying the estimate

$$
\sum_{j+|\beta|}\left\|\partial_{t}^{j} \partial_{x}^{\beta} u(t, x)\right\|_{L_{\text {loc }}^{2}\left(\mathbb{R}_{x^{\prime}}^{n-1} \times \overline{\mathbb{R}_{x_{n}}^{+}}\right)} \leq C_{\delta} \sum_{j+|\beta|}\left\|\partial_{t}^{j} \partial_{x}^{\beta} u(0, x)\right\|_{L_{\text {loc }}^{2}\left(\mathbb{R}_{x^{\prime}}^{n-1} \times \overline{\mathbb{R}_{x_{n}}^{+}}\right)}
$$

with a constant $C_{\delta}$ depending on $\delta$. Notice that (1.5) implies that the boundary $x_{n}=0$ is noncharacteristic for $\mathrm{P}\left(\mathrm{t}, x, \partial_{t}, \partial_{x}\right)$. So $\mathfrak{u}(\mathrm{t}, x) \in \mathcal{C}^{\infty}\left(\overline{\mathbb{R}_{x_{n}}^{+}} ; \mathrm{D}^{\prime}\left(\mathbb{R}^{n}\right)\right)$ (see Theorem B.2.9 in Hörmander [10]) and the trace $\mathfrak{u}_{\mid x_{n}=0}$ is meaningful. The same argument shows that $\nabla_{\chi} \mathfrak{u}(t,$.$) and \mathfrak{u}_{\mathfrak{t}}(t,$. depend continuously on $t$. Thus we obtain the existence and uniqueness of the solution of (1.4) in $\Omega \cap\{|t-s| \leq \epsilon\}$. We can determine $\epsilon>0$ uniformly with respect to $s$, provided $|s| \leq 2$. Making a construction by steps of length $\epsilon$, we cover the interval $|t-s| \leq D$ and the proof is complete.

Following Theorem 2, we can introduce the propagator $\mathcal{U}(t, s)$ defined by (1.6). Combining the results of Theorem 2 and the periodicity of $\mathrm{O}(\mathrm{t})$ and $\mathrm{a}(\mathrm{t}, \mathrm{x})$, we deduce the following.

Proposition 1. Assume (1.1), (1.2), (1.3) and (1.5) fulfilled. Then, we have

$$
\begin{gathered}
\mathcal{U}(\mathrm{t}+\mathrm{T}, \mathrm{s}+\mathrm{T})=\mathcal{U}(\mathrm{t}, \mathrm{s}), \\
\|\mathcal{U}(\mathrm{t}, \mathrm{s})\|_{\mathcal{L}(\mathrm{H}(\mathrm{s}), \mathrm{H}(\mathrm{t}))} \leq \mathrm{B} e^{\mathrm{A}|\mathrm{t}-\mathrm{s}|} .
\end{gathered}
$$

Proof. The proof of (2.5) is trivial. Let us show estimate (2.6). Applying (2.1), we obtain

$$
\sup _{|s|,|t| \leq T}\|\mathcal{U}(t, s)\|_{\mathcal{L}(H(s), H(t))}=\mathrm{C}<\infty
$$


Let $\mathrm{t}, \mathrm{s} \in \mathbb{R}$ and let $0 \leq \mathrm{t}^{\prime}, \mathrm{s}^{\prime}<\mathrm{T}$ be such that $\mathrm{t}=l \mathrm{~T}+\mathrm{t}^{\prime}$ and $s=k T+\mathrm{s}^{\prime}$ with $k, l \in \mathbb{Z}$. Then, applying (2.5), we obtain

$$
\mathcal{U}(\mathrm{t}, \mathrm{s})=\mathcal{U}\left(\mathrm{t}^{\prime}, 0\right) \mathcal{U}((\mathrm{k}-\mathrm{l}) \mathrm{T}, 0) \mathcal{U}\left(\mathrm{s}^{\prime}, 0\right)=\mathcal{U}\left(\mathrm{t}^{\prime}, 0\right) \mathcal{U}(\mathrm{T}, 0)^{\mathrm{k}-\mathrm{l}} \mathcal{U}\left(\mathrm{s}^{\prime}, 0\right)
$$

It follows that

$$
\|\mathcal{U}(t, s)\|_{\mathcal{L}(H(s), H(t))} \leq C^{2}(1+C)^{|k-l|} \leq C^{2} e^{\ln (1+C)|k-l|} \leq C^{2} e^{\ln (1+C)|t-s|}
$$

and we obtain (2.6) with $A=\ln (1+C)$.

Notice that, combing the arguments used in the proof of Theorem 2 with estimate (2.6), we can show that the Duhamel's principal holds. Let $\mathrm{P}_{1}$ and $\mathrm{P}_{2}$ be the projectors of $\mathbb{C}^{2}$ defined by

$$
P_{1}(h)=h_{1}, \quad P_{2}(h)=h_{2}, \quad h=\left(h_{1}, h_{2}\right) \in \mathbb{C}^{2}
$$

and let $\mathrm{P}^{1}, \mathrm{P}^{2} \in \mathcal{L}\left(\mathbb{C}, \mathbb{C}^{2}\right)$ be defined by

$$
P^{1}(h)=(h, 0), \quad P^{2}(h)=(0, h), \quad h \in \mathbb{C} .
$$

Denote by $\mathrm{V}(\mathrm{t}, \mathrm{s}): \mathrm{L}^{2}(\Omega(\mathrm{s})) \rightarrow \dot{\mathrm{H}}^{1}(\Omega(\mathrm{t}))$ the operator defined by

$$
V(t, s)=P_{1} \mathcal{U}(t, s) P^{2}
$$

Notice that for $\mathrm{h} \in \mathrm{L}^{2}(\Omega(\mathrm{s})), w=\mathrm{V}(\mathrm{t}, \mathrm{s}) \mathrm{h}$ is the solution of

$$
\left\{\begin{aligned}
\partial_{\mathrm{t}}^{2}(w)-\operatorname{div}_{\mathrm{x}}\left(\mathrm{a}(\mathrm{t}, x) \nabla_{\mathrm{x}} w\right) & =0, \\
w_{\mid \partial \Omega} & =0, \\
\left(w, \partial_{\mathrm{t}} w\right)_{\mid \mathrm{t}=\mathrm{s}} & =(0, \mathrm{~h}) .
\end{aligned}\right.
$$

Let $g(t, x)$ be a function defined on $\Omega$ such that, for $A_{1}>A$ (with $A$ the constant of (2.6)), $e^{-A_{1} t} g(t, x) \in L^{2}(\Omega)$ and $g(t, x)=0$ for $|x| \geq b$ with $b \geq \rho+1$. Then there exists a unique solution $v$ of

$$
\left\{\begin{aligned}
\partial_{t}^{2}(v)-\operatorname{div}_{x}\left(a(t, x) \nabla_{x} v\right) & =g(t, x), \\
v_{\mid \partial \Omega} & =0 \\
\left(v, \partial_{t} v\right)_{\mid t=s} & =(0,0) .
\end{aligned}\right.
$$

Moreover, this solution can be written in the following way

$$
v(t, .)=\int_{s}^{t} V(t, \tau) g(\tau, .) d \tau .
$$




\section{The meromorphic continuation of the cut-off resolvent $R_{\psi_{1}, \psi_{2}}(\theta)$}

The goal of this section is to prove the meromorphic continuation of $R_{\psi_{1}, \psi_{2}}(\theta)$, assuming (H1) fulfilled. The main result of this section is the following.

Theorem 3. Assume (H1), (1.1), (1.2), (1.3) and (1.5) fulfilled. Let $\psi_{1}, \psi_{2} \in \mathcal{C}_{0}^{\infty}\left(\mathbb{R}^{n}\right)$. Then, $R_{\psi_{1}, \psi_{2}}(\theta)$ admits a meromorphic continuation from $\{\theta \in \mathbb{C}: \mathfrak{I}(\theta)>A T\}$ to $\mathbb{C}$ for $n \geq 3$ odd and to $\mathbb{C}^{\prime}=\left\{\theta \in \mathbb{C}: \theta \notin 2 \pi \mathbb{Z}+i \mathbb{R}^{-}\right\}$for $n \geq 4$ even. Moreover, for $n \geq 4$ even, there exists $\epsilon_{0}>0$ such that, for $|\theta| \leq \epsilon_{0}$, we have

$$
R_{\psi_{1}, \psi_{2}}(\theta)=\sum_{k \geq-m} \sum_{j \geq-m_{k}} R_{k j} \theta^{k}(\log \theta)^{-j}
$$

Here $R_{k, j} \in \mathcal{L}(H(0))$ and, for $k<0$ or $j>0, R_{k, j}$ is a finite rank operator.

To prove Theorem 4, we will use some results of [26] and [13]. For this purpose, we introduce some tools and definitions of [26].

Let $\gamma \in \mathcal{C}^{\infty}(\mathbb{R})$ be such that $\gamma(t)=1$ for $t \geq-\frac{2 T}{3}-\frac{T}{10}$ and $\gamma(t)=0$ for $t \leq-\frac{2 T}{3}-\frac{2 T}{10}$. Set

$$
\mathrm{V}_{1}(\mathrm{t}, \mathrm{s})=\gamma(\mathrm{t}-\mathrm{s}) \mathrm{V}(\mathrm{t}, \mathrm{s}) .
$$

We recall that the Fourier-Bloch-Gelfand transform F is defined by

$$
\mathrm{F}(\phi)(\mathrm{t}, \theta)=\sum_{k=-\infty}^{+\infty}\left(\phi(\mathrm{t}+\mathrm{kT}, \cdot) \mathrm{e}^{\mathrm{ik} \theta}\right), \quad \phi \in \mathcal{C}_{0}^{\infty}\left(\mathbb{R} \times \mathbb{R}^{\mathrm{n}}\right) .
$$

Applying (2.6), for $\mathfrak{I}(\theta)>A T$, with $A>0$ the constant of (2.6), we can define

$$
F\left(\chi_{1} V_{1}(t, s) \chi_{2}\right)(t, \theta)=\sum_{k=-\infty}^{+\infty}\left(\chi_{1} V_{1}(t+k T, s) \chi_{2} e^{i k \theta}\right), \quad \chi_{1}, \chi_{2} \in \mathcal{C}_{0}^{\infty}\left(\mathbb{R}^{n}\right)
$$

and

$$
F^{\prime}\left(\chi_{1} V_{1}(t, s) \chi_{2}\right)(t, \theta)=e^{i \frac{t \theta}{T}} F\left(\chi_{1} V_{1}(t, s) \chi_{2}\right)(t, \theta), \quad \chi_{1}, \chi_{2} \in \mathcal{C}_{0}^{\infty}\left(\mathbb{R}^{n}\right)
$$

We will use the following definition of meromorphic continuation of a family of bounded operators.

Definition 1. Let $\mathrm{H}_{1}$ and $\mathrm{H}_{2}$ be Hilbert spaces. A family of bounded operators $\mathrm{Q}(\mathrm{t}, \mathrm{s}, \theta): \mathrm{H}_{1} \rightarrow \mathrm{H}_{2}$ is said to be meromorphic with respect to $\theta$ in a domain $\mathrm{D} \subset \mathbb{C}$, if $\mathrm{Q}(\mathrm{t}, \mathrm{s}, \theta)$ is meromorphically dependent on $\theta$ for $\theta \in \mathrm{D}$ and for any pole $\theta=\theta_{0}$ the coefficients of the negative powers of $\theta-\theta_{0}$ in the appropriate Laurent extension are finite-rank operators.

Denote $\mathbb{C}^{\prime}=\{z \in \mathbb{C}: z \neq 2 k \pi-i \mu, k \in \mathbb{Z}, \mu \geq 0\}$ and consider the following meromorphic continuation. 
Definition 2. We say that the family of operators $\mathrm{Q}(\mathrm{t}, \mathrm{s}, \theta)$, which are $\mathcal{C}^{\infty}$ with respect to $\mathrm{t}$ and $\mathrm{s}$, for $\mathrm{t} \in \mathbb{R}$ and $0 \leq \mathrm{s} \leq \frac{2 \mathrm{~T}}{3}$, and $\mathrm{T}$-periodic with respect to $\mathrm{t}$, has the property $\left(\mathrm{S}^{\prime}\right)$ if: 1) for odd $\mathrm{n}$ the operators $\mathrm{Q}(\mathrm{t}, \mathrm{s}, \theta), \theta \in \mathbb{C}$, and its derivatives with respect to $\mathrm{t}$ form a finitely-meromorphic family; 2) For even $\mathrm{n}$ the operators $\mathrm{Q}(\mathrm{t}, \mathrm{s}, \theta)$ and its derivatives with respect to $\mathrm{t}$ form a finitelymeromorphic family for $\theta \in \mathbb{C}^{\prime}$. Moreover, in a neighborhood of $\theta=0$ in $\mathbb{C}^{\prime}, \mathrm{Q}(\mathrm{t}, \mathrm{s}, \theta)$ has the form

$$
\mathrm{Q}(\mathrm{t}, \mathrm{s}, \theta)=\theta^{-\mathrm{m}} \sum_{j \geq 0}\left(\frac{\theta}{R_{t, s}(\log \theta)}\right)^{j} \mathrm{P}_{j, t, s}(\log \theta)+\mathrm{C}(\mathrm{t}, \mathrm{s}, \theta),
$$

where $\mathrm{C}(\mathrm{t}, \mathrm{s}, \theta)$ is analytic with respect to $\theta, \mathrm{R}_{\mathrm{t}, \mathrm{s}}$ is a polynomial, the $\mathrm{P}_{\mathrm{j}, \mathrm{t}, \mathrm{s}}$ are polynomials of order at most $\boldsymbol{l}_{\mathfrak{j}}$ and $\log$ is the logarithm defined on $\mathbb{C} \backslash \mathfrak{i} \mathbb{R}^{-}$. Moreover, $\mathrm{C}(\mathrm{t}, \mathrm{s}, \theta)$ and the coefficients of the polynomials $\mathrm{R}_{\mathrm{t}, \mathrm{s}}$ and $\mathrm{P}_{\mathrm{j}, \mathrm{t}, \mathrm{s}}$ are $\mathcal{C}^{\infty}$ and $\mathrm{T}$-periodic with respect to $\mathrm{t}$ and $\mathcal{C}^{\infty}$ with respect to $\mathrm{s}$ for $0 \leq \mathrm{s} \leq \frac{2 \mathrm{~T}}{3}$

Remark 2. Notice that if $Q(t, s, \theta)$ satisfies $\left(S^{\prime}\right)$ then $\partial_{t} Q(t, s, \theta)$ satisfies also $\left(S^{\prime}\right)$.

In 26] Vainberg proposed a general approach to problems with time-periodic perturbations including potentials, moving obstacles and high order operators, provided that the perturbations are non-trapping. One of the main results of [26] is the following.

Theorem 4. (Theorem 10, [26]) Assume that the mixed problem (1.4) is well posed, the Duhamel's principal holds and let (2.6) and (H1) be fulfilled. Then, for all $b \geq \rho+1$, there exists $T_{2}(b)>T_{1}(b)$ and an operator

$$
\mathrm{R}(\mathrm{t}, \mathrm{s}): \mathrm{L}^{2}(\Omega(\mathrm{s})) \rightarrow \dot{\mathrm{H}}^{1}(\Omega(\mathrm{t}))
$$

such that the following conditions are fulfilled:

(i) $R(t+T, s+T)=R(t, s)$,

(ii) $\mathrm{R}(\mathrm{t}, \mathrm{s})$ is bounded,

(iii) for all $\chi_{1}, \chi_{2} \in \mathcal{C}_{0}^{\infty}(|x| \leq b), F^{\prime}\left(\chi_{1} R(t, s) \chi_{2}\right)(t, \theta)$ admits a meromorphic continuation to the lower half plane satisfying property $\left(S^{\prime}\right)$ and $\chi_{1} R(t, s) \chi_{2}=\chi_{1} V(t, s) \chi_{2}$ for $t-s \geq T_{2}(b)$.

In [26] Vainberg established the result of Theorem 4 for $s=0$. In 13 it has been proven that this result can be generalized to $0 \leq s \leq \frac{2 T}{3}$. Combining these results with the properties established in Section 1, we obtain a meromorphic continuation of the Fourier-Bloch-Gelfand transform of the solutions of (1.4) with initial data $(0, g)$ and their derivatives of order 1 with respect to $t$.

Lemma 3.1. Assume (H1), (1.1), (1.2), (1.3) and (1.5) fulfilled. Then, for all $\psi_{1}, \psi_{2} \in \mathcal{C}_{0}^{\infty}\left(\mathbb{R}^{n}\right)$ and all $0 \leq s \leq \frac{2 T}{3}$,

$$
F^{\prime}\left(\psi_{1} V_{1}(t, s) \psi_{2}\right)(T, \theta) \quad \text { and } \quad F^{\prime}\left(\psi_{1} \partial_{t} V_{1}(t, s) \psi_{2}\right)(T, \theta)
$$

admit a meromorphic continuation with respect to $\theta$, continuous with respect to $s \in\left[0, \frac{2 \mathrm{~T}}{3}\right]$, from $\{\theta \in \mathbb{C}: \Im(\theta)>A T\}$ to $\mathbb{C}$ for $n \geq 3$ odd and to $\mathbb{C}^{\prime}=\left\{\theta \in \mathbb{C}: \theta \notin 2 \pi \mathbb{Z}+i \mathbb{R}^{-}\right\}$for $n \geq 4$ even. 
Moreover, for $n \geq 4$ even, there exists $\epsilon_{0}>0$ such that, for $|\theta| \leq \epsilon_{0}$, we have

$$
\begin{aligned}
& F^{\prime}\left(\psi_{1} V_{1}(t, s) \psi_{2}\right)(T, \theta)=\sum_{k \geq-m} \sum_{j \geq-m_{k}} Q_{k j}(s) \theta^{k}(\log \theta)^{-j} \\
& F^{\prime}\left(\psi_{1} \partial_{t} V_{1}(t, s) \psi_{2}\right)(T, \theta)=\sum_{k \geq-m} \sum_{j \geq-m_{k}} S_{k j}(s) \theta^{k}(\log \theta)^{-j}
\end{aligned}
$$

Here $Q_{k j}(s), S_{k j}(s) \in \mathcal{L}(H(s), H(0))$ and are continuous with respect to $s$ for $0 \leq s \leq \frac{2 T}{3}$.

Proof. According to Section 1, the mixed problem (1.4) is well posed, the Duhamel's principal holds, and (2.6), (2.7) are fulfilled. Thus, we can apply the results of Theorem 4. Choose $b \geq$ $\rho+1$ such that $\operatorname{supp} \psi_{1} \cup \operatorname{supp} \psi_{2} \subset\{x:|x| \leq b\}$. Take $h_{b} \in \mathcal{C}^{\infty}(\mathbb{R})$ such that $h_{b}(t)=1$ for $t \geq T_{2}(b)+\frac{6 T}{5}$ and $h_{b}(t)=0$ for $t \leq T_{2}(b)+T$. Then, for all $0 \leq s \leq \frac{2 T}{3}$, statement (iii) of Theorem 4 implies

$$
h_{b}(t) \psi_{1} V_{1}(t, s) \psi_{2}=h_{b}(t) \psi_{1} R(t, s) \psi_{2} .
$$

Thus, $F^{\prime}\left(h_{b}(t) \psi_{1} V_{1}(t, s) \psi_{2}\right)(t, \theta)$ admits a meromorphic continuation satisfying property $\left(S^{\prime}\right)$. From now on, we assume that $T_{2}(b)=k_{0} T$ with $k_{0} \in \mathbb{N}$. For $\mathfrak{I}(\theta)>A T$ we have

$$
F^{\prime}\left(\psi_{1} V_{1}(t, s) \psi_{2}\right)(T, \theta)=F^{\prime}\left(h_{b}(t) \psi_{1} V_{1}(t, s) \psi_{2}\right)(T, \theta)+F^{\prime}\left[\left(1-h_{b}(t)\right) \psi_{1} V_{1}(t, s) \psi_{2}\right](T, \theta)
$$

Since $1-h_{b}(t)=0$ for $t \geq T_{2}(b)+\frac{6 T}{5}=\left(k_{0}+1\right) T+\frac{T}{5}$, for $\mathfrak{I}(\theta)>A T$, we get

$$
F^{\prime}\left[\left(1-h_{b}(t)\right) \psi_{1} V_{1}(t, s) \psi_{2}\right](T, \theta)=e^{i \theta}\left[\sum_{k=1}^{k_{0}+1}\left(\psi_{1} V(k T, s) \psi_{2} e^{i k \theta}\right)+\gamma(-s) \psi_{1} V(0, s) \psi_{2}\right]
$$

Thus, $F^{\prime}\left[\left(1-h_{b}(t)\right) \psi_{1} V_{1}(t, s) \psi_{2}\right](T, \theta)$ admits an analytic continuation to $\mathbb{C}$. Combining the meromorphic continuation of $F^{\prime}\left(h_{b}(t) \psi_{1} V_{1}(t, s) \psi_{2}\right)(T, \theta)$, the analytic continuation of $F^{\prime}\left[\left(1-h_{b}(t)\right) \psi_{1} V_{1}(t, s) \psi_{2}\right](T, \theta)$ and representation (3.5), we obtain the meromorphic continuation of $F^{\prime}\left(\psi_{1} V_{1}(t, s) \psi_{2}\right)(T, \theta)$. It remains to prove the meromorphic continuation of $F^{\prime}\left(\psi_{1} \partial_{t} V_{1}(t, s) \psi_{2}\right)(T, \theta)$. Notice that

$$
\partial_{t} V(t, s)=P_{2} \mathcal{U}(t, s) P^{2}
$$

and, for $\mathfrak{I}(\theta)>A T, F^{\prime}\left(\psi_{1} V_{1}(t, s)\right)(t, \theta)$ is well defined. For $\mathfrak{I}(\theta)>A T$, we have

$$
\begin{aligned}
\partial_{t}\left[F^{\prime}\left(h_{b}(t) \psi_{1} V_{1}(t, s) \psi_{2}\right)(t, \theta)\right]= & \frac{i \theta}{T} F^{\prime}\left(h_{b}(t) \psi_{1} V_{1}(t, s) \psi_{2}\right)(t, \theta)+F^{\prime}\left(h_{b}^{\prime}(t) \psi_{1} V_{1}(t, s) \psi_{2}\right)(t, \theta) \\
& +F^{\prime}\left(h_{b}(t) \psi_{1} \partial_{t} V_{1}(t, s)\right)(t, \theta)
\end{aligned}
$$

Thus, for $\mathfrak{I}(\theta)>$ AT, we get

$$
\begin{aligned}
F^{\prime}\left(h_{b}(t) \psi_{1} \partial_{t} V_{1}(t, s) \psi_{2}\right)(t, \theta)= & \partial_{t}\left[F^{\prime}\left(h_{b}(t) \psi_{1} V_{1}(t, s) \psi_{2}\right)(t, \theta)\right]-F^{\prime}\left(h_{b}^{\prime}(t) \psi_{1} V_{1}(t, s) \psi_{2}\right)(t, \theta) \\
& -\frac{i \theta}{T} F^{\prime}\left(h_{b}(t) \psi_{1} V_{1}(t, s) \psi_{2}\right)(t, \theta)
\end{aligned}
$$


Since $F^{\prime}\left(h_{b}(t) \psi_{1} V_{1}(t, s) \psi_{2}\right)(t, \theta)$ admits a meromorphic continuation satisfying property $\left(S^{\prime}\right)$

$$
\partial_{t}\left[F^{\prime}\left(h_{b}(t) \psi_{1} V_{1}(t, s) \psi_{2}\right)(t, \theta)\right] \text { and } \frac{i \theta}{T} F^{\prime}\left(h_{b}(t) \psi_{1} V_{1}(t, s) \psi_{2}\right)(t, \theta)
$$

admit also a meromorphic continuation satisfying property $\left(S^{\prime}\right)$. Moreover, since $h_{b}^{\prime}(t)=0$ for $t \geq T_{2}(b)+\frac{6 T}{5}, F^{\prime}\left(h_{b}^{\prime}(t) \psi_{1} V_{1}(t, s) \psi_{2}\right)(t, \theta)$ admits an analytic continuation with respect to $\theta$. It follows that $F^{\prime}\left(h_{b}(t) \psi_{1} \partial_{t} V_{1}(t, s) \psi_{2}\right)(t, \theta)$ admits a meromorphic continuation satisfying property $\left(\mathrm{S}^{\prime}\right)$. We conclude by repeating the arguments used for proving the meromorphic continuation of $F^{\prime}\left(\psi_{1} V_{1}(t, s) \psi_{2}\right)(T, \theta)$.

Consider the operator defined by

$$
\mathrm{U}(\mathrm{t}, \mathrm{s})=\mathrm{P}_{1} \mathcal{U}(\mathrm{t}, \mathrm{s}) \mathrm{P}^{1}
$$

For all $\mathrm{h} \in \dot{\mathrm{H}}^{1}(\Omega(\mathrm{s})), w=\mathrm{U}(\mathrm{t}, \mathrm{s}) \mathrm{h}$ is the solution of

$$
\left\{\begin{aligned}
\partial_{\mathrm{t}}^{2} w-\operatorname{div}_{\chi}\left(\mathrm{a}(\mathrm{t}, x) \nabla_{x} w\right) & =0, \\
w_{\mid \partial \Omega} & =0, \\
\left(w, w_{\mathrm{t}}\right)_{\mid \mathrm{t}=\mathrm{s}} & =(\mathrm{h}, 0) .
\end{aligned}\right.
$$

Let $\gamma_{1} \in \mathcal{C}^{\infty}(\mathbb{R})$ be such that $\gamma_{1}(t)=1$ for $t \geq-\frac{T}{20}$ and $\gamma_{1}(t)=0$ for $t \leq-\frac{T}{10}$. Set

$$
\mathrm{U}_{1}(\mathrm{t}, \mathrm{s})=\gamma_{1}(\mathrm{t}-\mathrm{s}) \mathrm{u}(\mathrm{t}, \mathrm{s})
$$

Applying (2.6), for $\Im(\theta)>A T$ and $\psi_{1} \psi_{2} \in \mathcal{C}_{0}^{\infty}\left(\mathbb{R}^{n}\right)$ we can define $F^{\prime}\left(\psi_{1} U_{1}(t, s) \psi_{2}\right)(t, \theta)$ and $F^{\prime}\left(\psi_{1} \partial_{t} U_{1}(t, s) \psi_{2}\right)(t, \theta)$. From the results of Lemma 1 we obtain the following meromorphic continuation of $F^{\prime}\left(\psi_{1} U_{1}(t, s) \psi_{2}\right)(T, \theta)$ and $F^{\prime}\left(\psi_{1} \partial_{t} U_{1}(t, s) \psi_{2}\right)(T, \theta)$.

Lemma 3.2. Assume (H1), (1.1), (1.2), (1.3) and (1.5) fulfilled. Then, for all $\psi_{1}, \psi_{2} \in \mathcal{C}_{0}^{\infty}\left(\mathbb{R}^{n}\right)$,

$$
F^{\prime}\left(\psi_{1} U_{1}(t, s) \psi_{2}\right)(T, \theta) \quad \text { and } \quad F^{\prime}\left(\psi_{1} \partial_{t} U_{1}(t, s) \psi_{2}\right)(T, \theta)
$$

admit a meromorphic continuation with respect to $\theta$, continuous with respect to $s \in\left[0, \frac{2 T}{3}\right]$, from $\{\theta \in \mathbb{C}: \Im(\theta)>A T\}$ to $\mathbb{C}$ for $n \geq 3$ odd and to $\mathbb{C}^{\prime}$ for $n \geq 4$ even. Moreover, for $n \geq 4$ even, there exists $\epsilon_{0}>0$ such that, for $|\theta| \leq \epsilon_{0}$, we have

$$
\begin{aligned}
& F^{\prime}\left(\psi_{1} \mathrm{U}_{1}(t, 0) \psi_{2}\right)(T, \theta)=\sum_{k \geq-m} \sum_{j \geq-m_{k}} M_{k j} \theta^{k}(\log \theta)^{-j} . \\
& F^{\prime}\left(\psi_{1} \partial_{t} u_{1}(t, 0) \psi_{2}\right)(T, \theta)=\sum_{k \geq-m} \sum_{j \geq-m_{k}} N_{k j} \theta^{k}(\log \theta)^{-j} .
\end{aligned}
$$

Here $M_{k j}, N_{k j} \in \mathcal{L}(H(0))$ and, for $k<0$ or $j>0, M_{k j}, N_{k j}$ are a finite rank operator. 
Proof. Let $\alpha \in \mathcal{C}^{\infty}(\mathbb{R})$ be such that $\alpha(t)=0$ for $t \leq \frac{T}{2}$ and $\alpha(t)=1$ for $t \geq \frac{2 T}{3}$. For all $h \in \dot{H}^{1}(\Omega(0)), Z=\alpha(t) U(t, 0) h$ is the solution of

$$
\left\{\begin{aligned}
\partial_{\mathrm{t}}^{2} \mathrm{Z}-\operatorname{div}_{x}\left(\mathrm{a}(\mathrm{t}, x) \nabla_{x} \mathrm{Z}\right) & =\left[\partial_{\mathrm{t}}^{2}, \alpha\right](\mathrm{t}) \mathrm{U}(\mathrm{t}, 0) \mathrm{h}, \\
\mathrm{Z}_{\mid \partial \Omega} & =0 \\
\left(\mathrm{Z}, \partial_{\mathrm{t}} \mathrm{Z}\right)_{\mid \mathrm{t}=0} & =(0,0) .
\end{aligned}\right.
$$

We deduce from the Cauchy problem (3.9) the following representation

$$
\mathrm{U}(\mathrm{t}, 0)=\alpha(\mathrm{t}) \mathrm{U}(\mathrm{t}, 0)=\int_{0}^{\mathrm{t}} \mathrm{V}(\mathrm{t}, \mathrm{s})\left[\partial_{\mathrm{t}}^{2}, \alpha\right](\mathrm{s}) \mathrm{U}(\mathrm{s}, 0) \mathrm{ds}, \quad \mathrm{t} \geq \mathrm{T} .
$$

Since $\left[\partial_{t}^{2}, \alpha\right](t)=0$ for $t>\frac{2 T}{3}$, the formula (3.10) becomes

$$
\mathrm{U}(\mathrm{t}, 0)=\int_{0}^{\frac{2 \mathrm{~T}}{3}} \mathrm{~V}(\mathrm{t}, \mathrm{s})\left[\partial_{\mathrm{t}}^{2}, \alpha\right](s) \mathrm{U}(\mathrm{s}, 0) \mathrm{d} s, \quad \mathrm{t} \geq \mathrm{T} .
$$

Let $R>0$ be such that

$$
\operatorname{supp} \psi_{1} \cup \operatorname{supp} \psi_{2} \subset\{x:|x| \leq R\} .
$$

Choose $\mathrm{b}=\mathrm{R}+\rho+\mathrm{T}+1$ and take $\chi \in \mathcal{C}_{0}^{\infty}(|x| \leq \mathrm{b})$ such that

$$
\chi(x)=1 \text { for }|x| \leq R+\rho+T .
$$

The finite speed of propagation implies

$$
\psi_{1} \mathrm{U}(\mathrm{t}, 0) \psi_{2}=\int_{0}^{\frac{2 T}{3}} \psi_{1} \mathrm{~V}(\mathrm{t}, \mathrm{s}) \chi\left[\partial_{\mathrm{t}}^{2}, \alpha\right](\mathrm{s}) \mathrm{U}(\mathrm{s}, 0) \psi_{2} \mathrm{ds}, \quad \mathrm{t} \geq \mathrm{T} .
$$

Thus, for $\mathfrak{I}(\theta)>A T$, we obtain

$$
\begin{aligned}
\mathrm{F}^{\prime}\left(\psi_{1} \mathrm{U}_{1}(\mathrm{t}, 0) \psi_{2}\right)(\mathrm{T}, \theta)= & \mathrm{F}^{\prime}\left[\int_{0}^{\frac{2 \mathrm{~T}}{3}} \psi_{1} \mathrm{~V}_{1}(\mathrm{t}, \mathrm{s}) \chi\left[\partial_{\mathrm{t}}^{2}, \alpha\right](\mathrm{s}) \mathrm{U}(\mathrm{s}, 0) \psi_{2} \mathrm{ds}\right](\mathrm{T}, \theta) \\
& -\int_{0}^{\frac{2 \mathrm{~T}}{3}} \psi_{1} \mathrm{~V}_{1}(0, \mathrm{~s}) \chi\left[\partial_{\mathrm{t}}^{2}, \alpha\right](\mathrm{s}) \mathrm{U}(\mathrm{s}, 0) \psi_{2} \mathrm{~d} s+e^{\mathrm{i} \theta} \psi_{1} \psi_{2}
\end{aligned}
$$

and it follows

$$
\begin{aligned}
F^{\prime}\left[\psi_{1} U_{1}(t, 0) \psi_{2}\right](T, \theta)= & \int_{0}^{\frac{2 T}{3}} F^{\prime}\left[\psi_{1} V_{1}(t, s) \chi\right](T, \theta)\left[\partial_{t}^{2}, \alpha\right](s) U(s, 0) \psi_{2} d s \\
& -\int_{0}^{\frac{2 T}{3}} \psi_{1} V_{1}(0, s) \chi\left[\partial_{t}^{2}, \alpha\right](s) \mathrm{U}(s, 0) \psi_{2} d s+e^{i \theta} \psi_{1} \psi_{2} .
\end{aligned}
$$

Combining this representation with the meromorphic continuation of $F^{\prime}\left(\psi_{1} V_{1}(t, s) \chi\right)(T, \theta)$ established in Lemma 1, we prove the meromorphic continuation of $F^{\prime}\left(\psi_{1} \mathrm{U}(\mathrm{t}, 0) \psi_{2}\right)(\mathrm{T}, \theta)$ as well as (3.7). 
It remains to prove the meromorphic continuation of $F^{\prime}\left(\psi_{1} \partial_{t} U(t, 0) \psi_{2}\right)(T, \theta)$. Let $\beta \in \mathcal{C}_{0}^{\infty}\left(\mathbb{R}^{n}\right)$. The formula (3.11) implies that, for $t \geq T$, we have

$$
\partial_{t} \mathrm{U}(\mathrm{t}, 0) \beta=\int_{0}^{\frac{2 \mathrm{~T}}{3}} \partial_{\mathrm{t}} \mathrm{V}(\mathrm{t}, \mathrm{s})\left[\partial_{\mathrm{t}}^{2}, \alpha\right](\mathrm{s}) \mathrm{U}(\mathrm{s}, 0) \beta \mathrm{d} s
$$

By density, this leads to

$$
\psi_{1} \partial_{t} \mathrm{U}(\mathrm{t}, 0) \psi_{2}=\int_{0}^{\frac{2 \mathrm{~T}}{3}} \psi_{1} \partial_{\mathrm{t}} \mathrm{V}(\mathrm{t}, \mathrm{s}) \chi\left[\partial_{\mathrm{t}}^{2}, \alpha\right](\mathrm{s}) \mathrm{U}(\mathrm{s}, 0) \psi_{2} \mathrm{ds}, \quad \mathrm{t} \geq \mathrm{T}
$$

and, for $\mathfrak{I}(\theta)>A T$, we get

$$
\begin{aligned}
F^{\prime}\left(\psi_{1} \partial_{t} U_{1}(t, 0) \psi_{2}\right)(T, \theta)= & \int_{0}^{\frac{2 T}{3}} F^{\prime}\left(\psi_{1} \partial_{t} V_{1}(t, s) \chi\right)(T, \theta)\left[\partial_{t}^{2}, \alpha\right](s) U(s, 0) \psi_{2} d s \\
& -\int_{0}^{\frac{2 T}{3}} \psi_{1} V_{1}(0, s) \chi\left[\partial_{t}^{2}, \alpha\right](s) U(s, 0) \psi_{2} d s .
\end{aligned}
$$

We conclude by combining this representation with the results of Lemma 1.

Proof of Theorem 4. By definition, we can write

$$
\gamma_{1}(t) \psi_{1} \mathcal{U}(t, 0) \psi_{2}=\left(\begin{array}{cc}
\gamma_{1}(t) \psi_{1} U(t, 0) \psi_{2} & \gamma_{1}(t) \psi_{1} V(t, 0) \psi_{2} \\
\gamma_{1}(t) \psi_{1} \partial_{t} U(t, 0) \psi_{2} & \gamma_{1}(t) \psi_{1} \partial_{t} V(t, 0) \psi_{2}
\end{array}\right) .
$$

Moreover, for $\mathfrak{I}(\theta)>A T$, we have

$$
F^{\prime}\left[\gamma_{1}(t) \psi_{1} \mathcal{U}(t, 0) \psi_{2}\right](T, \theta)=e^{i \theta} \sum_{k=0}^{\infty}\left(\psi_{1} \mathcal{U}(T+k T, 0) \psi_{2} e^{i k \theta}\right)=-e^{-i \theta} R_{\psi_{1}, \psi_{2}}(\theta)
$$

and we obtain

$$
\begin{aligned}
R_{\psi_{1}, \psi_{2}}(\theta) & =-e^{i \theta} F^{\prime}\left[\gamma_{1}(t) \psi_{1} \mathcal{U}(t, 0) \psi_{2}\right](T, \theta) \\
& =-\left(\begin{array}{cc}
e^{i \theta} F^{\prime}\left(\psi_{1} U_{1}(t, 0) \psi_{2}\right)(T, \theta) & e^{i \theta} F^{\prime}\left(\psi_{1} V_{1}(t, 0) \psi_{2}\right)(T, \theta) \\
e^{i \theta} F^{\prime}\left(\psi_{1} \partial_{t} U_{1}(t, 0) \psi_{2}\right)(T, \theta) & e^{i \theta} F^{\prime}\left(\psi_{1} \partial_{t} V_{1}(t, 0) \psi_{2}\right)(T, \theta)
\end{array}\right) .
\end{aligned}
$$

Thus, combining the results of Lemma 1 and Lemma 2, we prove Theorem 4.

\section{Local energy decay}

The goal of this section is to prove Theorem 1, assuming (H1) and (H2) fulfilled. For this purpose, we show how assumption (H2) alter the meromorphic continuation of $R_{\psi_{1}, \psi_{2}}(\theta)$ established in Section 2. Then, by integrating on a suitable contour, we prove the local energy decay. We treat separately the case of odd and even dimensions. We start with $n$ odd. 
Lemma 4.1. Assume $\mathrm{n} \geq 3$ odd, (1.1), (1.2), (1.3), (1.5), (H1) and (H2) fulfilled. Then, for all $\psi_{1}, \psi_{2} \in \mathcal{C}_{0}^{\infty}(|x| \leq \rho+1)$, we get

$$
\left\|\psi_{1} \mathcal{U}(\mathrm{t}, \mathrm{s}) \psi_{2}\right\|_{\mathcal{L}(\mathrm{H}(\mathrm{s}), \mathrm{H}(\mathrm{t}))} \leq \mathrm{C} e^{-\delta(\mathrm{t}-\mathrm{s})}, \quad \mathrm{t} \geq \mathrm{s} .
$$

Proof. Notice that, for $\mathfrak{I}(\theta)>A T, F^{\prime}\left[\gamma_{1}(t) \phi_{1} \mathcal{U}(t, 0) \phi_{2}\right](t, \theta)$ is T-periodic with respect to $t$ and $2 \pi$-periodic with respect to $\theta$ (see [26] Theorem ). Applying (3.12), we get

$$
F^{\prime}\left[\gamma_{1}(t) \phi_{1} \mathcal{U}(t, 0) \phi_{2}\right](d T, \theta)=F^{\prime}\left[\gamma_{1}(t) \phi_{1} \mathcal{U}(t, 0) \phi_{2}\right](T, \theta)=-e^{-i \theta} R_{\phi_{1}, \phi_{2}}(\theta) .
$$

Moreover, from [26] we have the following inversion formula (see Lemma 1 of [26])

$$
\phi_{1} \mathcal{U}(\mathrm{dT}, 0) \phi_{2}=\frac{1}{2 \pi} \int_{[i(\mathrm{~A}+1) \mathrm{T}-\pi, \mathrm{i}(\mathrm{A}+1) \mathrm{T}+\pi]} \underset{e^{-i \mathrm{~d} \theta} \mathrm{F}^{\prime}\left[\gamma_{1}(\mathrm{t}) \phi_{1} \mathcal{U}(\mathrm{t}, 0) \phi_{2}\right](\mathrm{T}, \theta) \mathrm{d} \theta}{ }
$$

We will show (4.1), by combining these statements with assumption (H2).

First, assumption (H2) and (4.2) imply that $\mathrm{F}^{\prime}\left[\gamma_{1}(\mathrm{t}) \phi_{1} \mathcal{U}(\mathrm{t}, 0) \phi_{2}\right](\mathrm{dT}, \theta)$ has no poles on $\{\theta: \mathfrak{I}(\theta \geq 0)\}$. It follows that there exists $\delta>0$ such that $\mathrm{F}^{\prime}\left[\gamma_{1}(\mathrm{t}) \phi_{1} \mathcal{U}(\mathrm{t}, 0) \phi_{2}\right](\mathrm{dT}, \theta)$ has no poles on $\{\theta: \Im(\theta) \geq-\delta \mathrm{T},-\pi \leq \operatorname{Re}(\theta) \leq \pi\}$. Consider the contour $\mathrm{C}_{1}$ defined by

$\mathcal{C}_{1}=[i(A+1) T+\pi, \mathfrak{i}(A+1) T-\pi] \cup[i(A+1) T-\pi,-i \delta T-\pi] \cup[-i \delta T-\pi,-i \delta T+\pi] \cup[-i \delta T+\pi, i(A+1) T+\pi]$.

The Cauchy formula implies

$$
\int_{C_{1}} e^{-i d \theta} F^{\prime}\left[\gamma_{1}(t) \phi_{1} \mathcal{U}(t, 0) \phi_{2}\right](T, \theta) \mathrm{d} \theta=0 .
$$

Also, since $F^{\prime}\left[\gamma_{1}(t) \phi_{1} \mathcal{U}(t, 0) \phi_{2}\right](T, \theta)$ is $2 \pi$-periodic with respect to $\theta$ we have

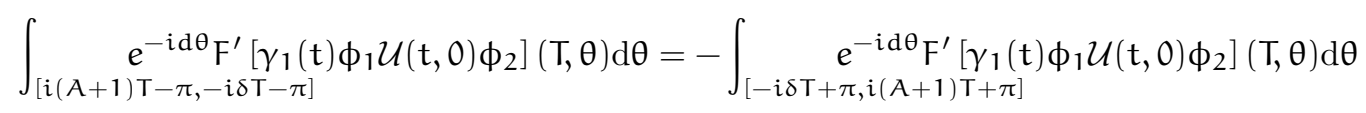

and we obtain

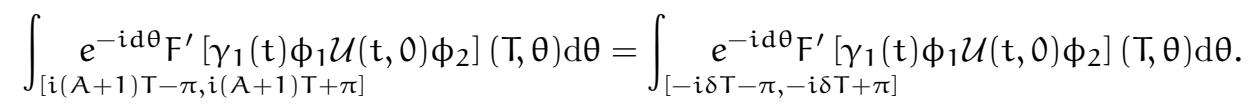

It is obvious that

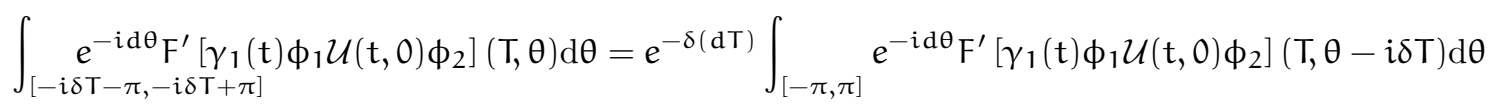

and combining this with (4.4) and the inversion formula (4.3), we get

$$
\left\|\phi_{1} \mathcal{U}(\mathrm{dT}, 0) \phi_{2}\right\|_{\mathcal{L}(\mathrm{H}(0))} \leq \mathrm{C} e^{-\delta(\mathrm{dT})} .
$$

Now let $\psi_{1}, \psi_{2} \in \mathcal{C}_{0}^{\infty}(|x| \leq \rho+1)$, and let $t, s \in \mathbb{R}$ be such that $t \geq s$. we write $t=t^{\prime}+m T$ and $s=s^{\prime}+k T$ with $0 \leq t^{\prime}, s^{\prime}<T$ and $m, k \in \mathbb{N}$. The finite speed of propagation implies

$$
\psi_{1} \mathcal{U}(\mathrm{t}, \mathrm{s}) \psi_{2}=\psi_{1} \mathcal{U}\left(\mathrm{t}^{\prime}, 0\right) \phi_{1} \mathcal{U}((\mathrm{m}-\mathrm{k}) \mathrm{T}, 0) \phi_{2} \mathcal{U}\left(0, \mathrm{~s}^{\prime}\right) \psi_{2}
$$


Then, applying (4.5) and Theorem 2, we obtain

$$
\left\|\psi_{1} \mathcal{U}(\mathrm{t}, \mathrm{s}) \psi_{2}\right\|_{\mathcal{L}(\mathrm{H}(\mathrm{s}), \mathrm{H}(\mathrm{t}))} \leq \mathrm{C}\left\|\phi_{1} \mathcal{U}((\mathrm{m}-\mathrm{k}) \mathrm{T}, 0) \phi_{2}\right\|_{\mathcal{L}(\mathrm{H}(\mathrm{o}))} \leq \mathrm{C}^{\prime} \mathrm{e}^{-\delta((\mathrm{m}-\mathrm{k}) \mathrm{T})} \leq \mathrm{C}^{\prime} \mathrm{e}^{-\delta(\mathrm{t}-\mathrm{s})} .
$$

Lemma 4.2. Assume $\mathrm{n} \geq 4$ even, (1.1), (1.2), (1.3), (1.5), (H1) and (H2) fulfilled. Then, for all $\psi_{1}, \psi_{2} \in \mathcal{C}_{0}^{\infty}(|x| \leq \rho+1)$, we get

$$
\left\|\psi_{1} \mathcal{U}(\mathrm{t}, \mathrm{s}) \psi_{2}\right\|_{\mathcal{L}(\mathrm{H}(\mathrm{s}), \mathrm{H}(\mathrm{t}))} \leq \mathrm{Cp}(\mathrm{t}-\mathrm{s}), \quad \mathrm{t} \geq \mathrm{s}
$$

with

$$
p(t)=\frac{1}{(t+1) \ln ^{2}(t+e)} .
$$

Proof. Repeating the arguments used in the proof of Lemma 3 , we obtain that $\mathrm{F}^{\prime}\left[\gamma_{1}(\mathrm{t}) \phi_{1} \mathcal{U}(\mathrm{t}, 0) \phi_{2}\right](\mathrm{T}, \theta)$ has no poles on $\left\{\theta \in \mathbb{C}^{\prime}: \operatorname{Im}(\theta) \geq 0\right\}$. Moreover, representation (3.1) implies that there exists $\epsilon_{0}>0$ such that for $\theta \in \mathbb{C}^{\prime}$ with $|\theta| \leq \epsilon_{0}$ we have

$$
F^{\prime}\left[\gamma_{1}(t) \phi_{1} \mathcal{U}(t, 0) \phi_{2}\right](T, \theta)=\sum_{k \geq-m} \sum_{j \geq-m_{k}} R_{k j} \theta^{k}(\log \theta)^{-j}
$$

and assumption (H2) implies that in this representation we have $R_{k j}=0$ for $k<0$ or $k=0$ and $j<0$. It follows that, for $\theta \in \mathbb{C}^{\prime}$ with $|\theta| \leq \epsilon_{0}$, we obtain the following representation

$$
\mathrm{F}^{\prime}\left[\gamma_{1}(\mathrm{t}) \phi_{1} \mathcal{U}(\mathrm{t}, 0) \phi_{2}\right](\mathrm{T}, \theta)=A(\theta)+B \theta^{\mathrm{m}_{\mathrm{o}}} \log (\theta)^{-\mu}+\underset{\theta \rightarrow 0}{\mathrm{o}}\left(\theta^{\mathrm{m}_{0}} \log (\theta)^{-\mu}\right)
$$

with $A(\theta)$ analytic with respect to $\theta$ for $|\theta| \leq \epsilon_{0}$, B a finite-dimensional operator, $m_{0} \geq 0$ and $\mu \geq 1$. Since $F^{\prime}\left[\gamma_{1}(t) \phi_{1} \mathcal{U}(t, 0) \phi_{2}\right](T, \theta)$ has no poles on $\left\{\theta \in \mathbb{C}^{\prime}: \operatorname{Im}(\theta) \geq 0\right\}$, there exists $0<\delta \leq \frac{\epsilon_{0}}{T}$ and $0<v<\epsilon_{0}$ sufficiently small such that $F^{\prime}\left[\gamma_{1}(t) \phi_{1} \mathcal{U}(t, 0) \phi_{2}\right](T, \theta)$ has no poles on

$$
\{\theta \in \mathbb{C}: \operatorname{Im}(\theta) \geq-\delta T,-\pi \leq \operatorname{Re}(\theta) \leq-v, v \leq \operatorname{Re}(\theta) \leq \pi\}
$$

Consider the contour $\Sigma=\Gamma_{1} \cup \omega \cup \Gamma_{2}$ where $\Gamma_{1}=[-i \delta T-\pi,-i \delta T-v], \Gamma_{2}=[-i \delta+v,-i \delta+\pi]$. The contour $\omega$ of $\mathbb{C}$, is a curve connecting $-i \delta T-v$ and $-i \delta T+v$ symmetric with respect to the axis $\operatorname{Re}(\theta)=0$. The part of $\omega$ lying in $\{\theta: \operatorname{Im}(\theta) \geq 0\}$ is a half-circle with radius $v$, $\omega \cap\{\theta: \operatorname{Re}(\theta)<0, \operatorname{Im}(\theta) \leq 0\}=[-v-i \delta \mathrm{T},-v]$ and $\omega \cap\{\theta: \operatorname{Re}(\theta)>0, \operatorname{Im}(\theta) \leq 0\}=[v, v-i \delta \mathrm{T}]$. Thus, $\omega$ is included in the region where we have no poles of $F^{\prime}\left[\gamma_{1}(t) \phi_{1} \mathcal{U}(t, 0) \phi_{2}\right](T, \theta)$. Consider the closed contour

$$
\mathcal{C}_{2}=[i(A+1) T+\pi, i(A+1) T-\pi] \cup[i(A+1) T-\pi,-i \delta T-\pi] \cup \Sigma \cup[-i \delta T+\pi, i(A+1) T+\pi] .
$$

An application of the Cauchy formula yields

$$
\int_{\mathcal{C}_{2}} e^{-i \mathrm{~d} \theta} \mathrm{F}^{\prime}\left[\gamma_{1}(\mathrm{t}) \phi_{1} \mathcal{U}(\mathrm{t}, 0) \phi_{2}\right](\mathrm{T}, \theta) \mathrm{d} \theta=0
$$


Applying the same arguments as those used in the proof of Lemma 3, we obtain

$$
\int_{[i(A+1) T-\pi, i(A+1) T+\pi]} F\left[\gamma_{1}(t) \phi_{1} \mathcal{U}(t, 0) \phi_{2}\right](d T, \theta) d \theta=\int_{\Sigma} e^{-i d \theta} F^{\prime}\left[\gamma_{1}(t) \phi_{1} \mathcal{U}(t, 0) \phi_{2}\right](T, \theta) d \theta
$$

and the inversion formula (4.3) implies

$$
\phi_{1} \mathcal{U}(\mathrm{dT}, 0) \phi_{2}=\frac{1}{2 \pi} \int_{\Sigma} e^{-\mathrm{id} \theta} \mathrm{F}^{\prime}\left[\gamma_{1}(\mathrm{t}) \phi_{1} \mathcal{U}(\mathrm{t}, 0) \phi_{2}\right](\mathrm{T}, \theta) \mathrm{d} \theta, \quad \mathrm{d} \in \mathbb{N}
$$

Combining this representation with (4.8) and applying some arguments used in Lemma 2 and Lemma 3 of [13, we obtain (4.6).

Combining the results of Lemma 3 and Lemma 4, we prove Theorem 1.

\section{$5 \quad$ Examples of metrics $a(t, x)$ and obstacles $O(t)$}

In this section we will apply some properties of solutions of the wave equations with non-trapping metrics independent of $t$ and fixed obstacle to construct time periodic metrics and moving obstacles such that conditions (H1) and (H2) are fulfilled. For this purpose, we assume that (H1) is fulfilled for the metrics $a(t, x)$ and obstacle $\mathrm{O}(\mathrm{t})$ that we consider and we will establish examples for $(\mathrm{H} 2)$. In order to prove (H2), we will modify the size $T$ of the period of $a(t, x)$. This choice is justified by the properties of $\mathcal{U}(t, s)$.

Let $T_{1}>0$ and let $\left(\left(a_{T}(t, x), O_{T}(t)\right)\right)_{T \geq T_{1}}$ be a family of couples of functions and obstacles such that the following conditions are fulfilled:

(H3i) $a_{T}(t, x)$ and $\mathrm{O}_{\mathrm{T}}(\mathrm{t})$ are $\mathrm{T}$-periodic with respect to $t$ and $\mathrm{a}_{\mathrm{T}}(\mathrm{t}, \mathrm{x})$ satisfies (1.5),

(H3ii) for all $\mathrm{T} \geq \mathrm{T}_{1}$, if $(\mathrm{a}(\mathrm{t}, \mathrm{x}), \mathrm{O}(\mathrm{t}))=\left(\mathrm{a}_{\mathrm{T}}(\mathrm{t}, \mathrm{x}), \mathrm{O}_{\mathrm{T}}(\mathrm{t})\right)$ then conditions (1.1), (1.2), (1.3) and (H1) are fulfilled,

(H3iii) there exist a function $a_{1}(x)$ and an obstacle $\mathrm{O}$ independent of $t$ such that for

$$
(a(t, x), O(t))=\left(a_{1}(x), O\right)
$$

condition (H1) is fulfilled and, for all $T_{1} \leq t \leq T$, we have $a_{T}(t, x)=a_{1}(x)$ and $O_{T}(t)=O$.

Let $\mathrm{H}$ be the closure of the space $\mathcal{C}_{0}^{\infty}\left(\mathbb{R}^{n} \backslash \mathrm{O}\right) \times \mathcal{C}_{0}^{\infty}\left(\mathbb{R}^{n} \backslash \mathrm{O}\right)$ with respect to the norm

$$
\|f\|_{H}=\left(\int_{\mathbb{R}^{n} \backslash O}\left(\left|\nabla_{x} f_{1}\right|^{2}+\left|f_{2}\right|^{2}\right) d x\right)^{\frac{1}{2}}, \quad f=\left(f_{1}, f_{2}\right) \in \mathcal{C}_{0}^{\infty}\left(\mathbb{R}^{n} \backslash O\right) \times \mathcal{C}_{0}^{\infty}\left(\mathbb{R}^{n} \backslash O\right) .
$$


Consider the following Cauchy problem

$$
\left\{\begin{array}{c}
v_{\mathrm{tt}}-\operatorname{div}_{\chi}\left(\mathrm{a}_{1}(x) \nabla_{x} v\right)=0, \quad \mathrm{t} \in \mathbb{R}, \quad x \in \mathbb{R}^{\mathrm{n}} \backslash \mathrm{O} \\
v_{\mid \partial \mathrm{O}}=0, \quad \mathrm{t} \in \mathbb{R}, \\
\left(v, v_{\mathrm{t}}\right)(0)=\mathrm{f},
\end{array}\right.
$$

and the associate propagator

$$
\mathcal{V}(\mathrm{t}): \mathrm{H} \ni \mathrm{f} \longmapsto\left(v, v_{\mathrm{t}}\right)(\mathrm{t}) \in \mathrm{H} .
$$

Let $u$ be solution of (2.2). For $T_{1} \leq t \leq T$ we have

$$
\partial_{t}^{2} u-\operatorname{div}_{x}\left(a_{1}(x) \nabla_{x} u\right)=\partial_{t}^{2} u-\operatorname{div}_{x}\left(a_{T}(t, x) \nabla_{x} u\right)=0 .
$$

It follows that for $(a(t, x), O(t))=\left(a_{T}(t, x), O_{T}(t)\right)$ we get

$$
\mathcal{U}(\mathrm{t}, \mathrm{s})=\mathcal{V}(\mathrm{t}-\mathrm{s}), \quad \mathrm{T}_{1} \leq \mathrm{s}<\mathrm{t} \leq \mathrm{T}
$$

and

$$
\mathrm{H}(\mathrm{t})=\mathrm{H}, \quad \mathrm{T}_{1} \leq \mathrm{t} \leq \mathrm{T} .
$$

The asymptotic expansion of $\chi \mathcal{V}(t) \chi$ as $t \rightarrow+\infty$ has been studied by many authors (see [25, 24] and [28]). It has been proven that, for non-trapping metrics and for $n \geq 3$, the local energy decreases. To prove (H2), we will apply the following result.

Theorem 5. Assume $n \geq 3$. Let $\phi \in \mathcal{C}_{0}^{\infty}\left(\mathbb{R}^{n}\right)$. Then, we have

$$
\|\phi \mathcal{V}(\mathrm{t}) \phi\|_{\mathcal{L}(\mathrm{H})} \leq \mathrm{C}_{\phi} \mathrm{p}(\mathrm{t})
$$

with

$$
\left\{\begin{array}{l}
p(t)=e^{-\delta t} \text { for } n \text { odd } \\
p(t)=\langle t\rangle^{1-n} \text { for } n \text { even. }
\end{array}\right.
$$

Estimate (5.4) has been established by Vainberg in [24, 25] but also by Vodev in [27] and [28. For $n \geq 4$ even we will use the following identity.

Lemma 5.1. Let $\psi \in \mathcal{C}_{0}^{\infty}\left(|x| \leq \rho+1+\mathrm{T}_{1}\right)$ be such that $\psi=1$, for $|x| \leq \rho+\frac{1}{2}+\mathrm{T}_{1}$. Then, we have

$$
\mathcal{U}\left(T_{1}, 0\right)-\mathcal{V}\left(T_{1}\right)=\psi\left(\mathcal{U}\left(T_{1}, 0\right)-\mathcal{V}\left(T_{1}\right)\right)=\left(\mathcal{U}\left(T_{1}, 0\right)-\mathcal{V}\left(T_{1}\right)\right) \psi
$$

Proof. First, notice that (5.3) implies $\mathrm{H}(0)=\mathrm{H}$. Now, choose $\mathrm{g} \in \mathrm{H}(0)=\mathrm{H}$ and let $w$ be the function defined by $\left(w, w_{t}\right)(t)=\mathcal{U}(t, 0)(1-\psi) g$. The finite speed of propagation implies that, for $0 \leq \mathrm{t} \leq \mathrm{T}_{1}$ and $|x| \leq \rho+\frac{1}{2}$, we get $w(t, x)=0$. Moreover, we have

$$
\operatorname{div}_{x}\left(a_{1}(x) \nabla_{x}\right)=\Delta_{x}=\operatorname{div}_{x}\left(a(t, x) \nabla_{x}\right), \quad \text { for }|x|>\rho .
$$

Thus, $w$ is solution on $0 \leq t \leq T_{1}$ of the problem

$$
\left\{\begin{array}{c}
w_{\mathrm{tt}}-\operatorname{div}_{\chi}\left(\mathrm{a}_{1}(x) \nabla_{\mathrm{x}} w\right)=0, \quad \mathrm{t} \in \mathbb{R}, \quad x \in \mathbb{R}^{n} \backslash \mathrm{O} \\
w_{\mid \partial \mathrm{O}}=0, \quad \mathrm{t} \in \mathbb{R}, \\
\left(w, w_{\mathrm{t}}\right)(0)=(1-\psi) g
\end{array}\right.
$$


and it follows that

$$
\left(\mathcal{U}\left(T_{1}, 0\right)-\mathcal{V}\left(T_{1}\right)\right)(1-\psi)=0 .
$$

Now, let $u$ and $v$ be the functions defined by $\left(u, u_{t}\right)(t)=\mathcal{U}(t, 0) g$ and $\left(v, v_{t}\right)(t)=\mathcal{V}(t) g$ with $g \in \mathrm{H}$. Applying (5.6), we can easily show that on $(1-\psi) \mathfrak{u}$ is the solution of

$$
\left\{\begin{array}{c}
\left.\left.\partial_{\mathfrak{t}}^{2}((1-\psi) \mathfrak{u})\right)-\Delta_{x}((1-\psi) \mathfrak{u})\right)=\left[\Delta_{x}, \psi\right] \mathfrak{u}, \\
\left(((1-\psi) \mathfrak{u}),((1-\psi) \mathfrak{u})_{\mathfrak{t}}\right)(0)=(1-\psi) \mathrm{g},
\end{array}\right.
$$

and $(1-\psi) v$ is the solution of

$$
\left\{\begin{array}{c}
\left.\partial_{\mathrm{t}}^{2}(((1-\psi) v))-\Delta_{\mathrm{x}}((1-\psi) v)\right)=\left[\Delta_{\mathrm{x}}, \psi\right] v, \\
\left(((1-\psi) v),((1-\psi) v)_{\mathrm{t}}\right)(0)=(1-\psi) \mathrm{g} .
\end{array}\right.
$$

We have

$$
(1-\psi)\left(\mathcal{U}\left(T_{1}, 0\right)-\mathcal{V}\left(T_{1}\right)\right)=0 .
$$

Combining (5.7) and (5.8), we get (5.5).

Combining the arguments used in the proofs of Lemma 7, 8 and 9 and Theorem 14 of 13 . with the identity (5.5), we obtain the following.

Theorem 6. Assume $\mathrm{n} \geq 3$ and let $\left(\left(\mathrm{a}_{\mathrm{T}}(\mathrm{t}, \mathrm{x}), \mathrm{O}_{\mathrm{T}}(\mathrm{t})\right)\right)_{\mathrm{T} \geq \mathrm{T}_{1}}$ satisfy (H3i), (H3ii), (H3iii). Then, for $\mathrm{T}$ large enough and for $(a(t, x), O(t))=\left(a_{T}(t, x), O_{T}(t)\right)$, assumption (H2) is fulfilled.

Received: November 2011. Revised: November 2011.

\section{References}

[1] A. Bachelot and V. Petkov, Existence des opérateurs d'ondes pour les systèmes hyperboliques avec un potentiel périodique en temps, Ann. Inst. H. Poincaré (Physique théorique), 47 (1987), 383-428.

[2] C. O. Bloom and N. D. Kazarinoff, Energy decays locally even if total energy grows algebraically with time, J. Differential Equation, 16 (1974), 352-372.

[3] J-F. Bony and V. Petkov, Resonances for non trapping time-periodic perturbation, J. Phys. A: Math. Gen., 37 (2004), 9439-9449.

[4] N. Burq, Décroissance de l'énergie locale de l'équation des ondes pour le problme extérieur et absence de résonance au voisinage du réel, Acta Mathématica, 180 (1998), 1-29.

[5] N. Burq, Global Strichartz estimates for non-trapping geometries: About an article by H.Smith and C.Sogge, Commun. PDE, 28 (2003), 1675-1683.

[6] F. Colombini, V. Petkov and J. Rauch, Exponential growth for the wave equation with compact time-periodic positive potential, Comm. Pure Appl. Math., 62 (2009), 565-582. 
[7] F. Colombini and J. Rauch, Smooth localised parametric resonance for wave equations, J. Reine Angew. Math., 616 (2008), 1-14.

[8] J. Cooper and W. Strauss, Scattering theory of waves by periodically moving bodies, J. Funct. Anal., 47 (1982), 180-229.

[9] V. Georgiev and V. Petkov, RAGE theorem for power bounded operators and decay of local energy for moving obstacles, Ann. Inst. H. Poincaré Phys. Théor, 51 (1989), no.2, 155-185.

[10] L. Hörmander, The analysis of linear partial differential operators III, Springer-Verlag, 1985.

[11] Y. Kian, Strichartz estimates for the wave equation with a time-periodic non-trapping metric, Asymptotic Analysis, 68 (2010), 41-76.

[12] Y. Kian, Cauchy problem for semilinear wave equation with time-dependent metrics, Nonlinear Analysis, 73 (2010), 2204-2212.

[13] Y. Kian, Local energy decay in even dimensions for the wave equation with a time-periodic non-trapping metric and applications to Strichartz estimates, Serdica Math. J., 36 (2010), 329-370.

[14] R. Melrose, Singularities and energy decay in acoustical scattering, Duke Math. J., 46 (1979), 43-59.

[15] R. Melrose and J. Sjöstrand, Singularities of boundary value problem, Comm. Pure Appl. Math., I, 31 (1978), 593-617, II, 35 (1982), 129-168.

[16] C. Morawetz, J. Ralston, W. Strauss, Decay of solutions of wave equations outside nontrapping obstacle, Comm. Pure Appl. Math., 30 (1977), no.4, 447-508.

[17] J. L. Metcalfe, Global Strichartz estimates for solutions to the wave equation exterior to a convex obstacle, Trans. Amer. Math. Soc, 356 (2004), no.12, 4839-4855.

[18] S. Miyatake, Mixed problems for hyperbolic equation of second order, J. Math. Kyoto Univ., 13 (1973), 435-487.

[19] H. F. Smith and C. Sogge, Global Strichartz estimates for non-trapping perturbations of the Laplacian, Commun. PDE, 25 (2000), 2171-2183.

[20] V. Petkov, Scattering theory for hyperbolic operators, North Holland, Amsterdam, 1989.

[21] V. Petkov, Global Strichartz estimates for the wave equation with time-periodic potentials, J. Funct. Anal., 235 (2006), 357-376.

[22] G. Popov and Ts. Rangelov, Exponential growth of the local energy for moving obstacles, Osaka J. Math., 26 (1989), 881-895. 
[23] S-H. Tang and M. Zworski, Resonance expansions of scattered waves, Comm. Pure Appl. Math., 53 (2000), 1305-1334.

[24] B. Vainberg, On the short wave asymptotic behavior of solutions of stationary problems and the asymptotic behavior as $t \rightarrow \infty$ of solutions of nonstationary problems, Russian Math. Surveys, 30 (1975), 1-53.

[25] B. Vainberg, Asymptotic methods in Equation of mathematical physics, Gordon and Breach, New York, 1988.

[26] B. Vainberg, On the local energy of solutions of exterior mixed problems that are periodic with respect to $t$, Trans. Moscow Math. Soc. 1993, 191-216.

[27] G. Vodev, On the uniform decay of local energy, Serdica Math. J., 25 (1999), 191-206.

[28] G. Vodev, Local energy decay of solutions to the wave equation for non-trapping metrics, Ark. Math, 42 (2004), no 2, 379-397. 